\title{
TRACES OF HUMAN FUNCTIONAL ACTIVITY: MOMENT-TO-MOMENT FLUCTUATIONS IN FMRI DATA
}

\author{
Luca Dodero $^{1}$, Diego Sona ${ }^{1,4}$, Djalel E. Meskaldji ${ }^{2,3}$, Vittorio Murino ${ }^{1,5}$, Dimitri Van De Ville ${ }^{2,3}$ \\ ${ }^{1}$ Pattern Analysis and Computer Vision, PAVIS, Istituto Italiano di Tecnologia, Genova, Italy \\ ${ }^{2}$ Institute of Bioengineering, École Polytechnique Fédérale de Lausanne, Switzerland \\ ${ }^{3}$ Department of Radiology and Medical Informatics, University of Geneva, Switzerland \\ ${ }^{4}$ NeuroInformatics Laboratory, Fondazione Bruno Kessler, Trento, Italy \\ ${ }^{5}$ Dept. of Computer Science, University of Verona, Italy
}

\begin{abstract}
Dynamic functional connectivity (dFC) measured by functional magnetic resonance imaging (fMRI) shows evidence of large-scale networks with highly dynamic (re)configurations.

We propose a novel approach to extract traces of human brain function by the construction of a trajectory in a meaningful low-dimensional space. This allows studying dFC in more detail and identify possible meaningful brain states from the moment-to-moment fluctuations of the brain signals during resting state or naturalistic conditions such as passive movie watching. Specifically, we explored dynamic organization of sub-networks derived from the time-dependent graph Laplacian in combination with Riemannian manifold distance to measure dissimilarity over time of $\mathrm{dFC}$ and to subsequently build the trajectory of brain activity. As a proof-of-principle, we show results for an fMRI dataset containing both rest and movie epochs in 15 healthy participants. The movie condition varied (i.e., fearful, joyful, and neutral movie excerpts) and clearly influenced the subsequent resting-state period in terms of FC brain state.
\end{abstract}

Index Terms - Functional MRI, Riemannian distance, Network modeling, Brain activity

\section{INTRODUCTION}

Functional Magnetic Resonance Imaging (fMRI) is widely used to investigate brain function. Next to using evoked activity, many studies focus on resting state or naturalistic conditions to identify intrinsic functional networks [1]. One predominant measure to characterize interactions in restingstate fMRI (rs-fMRI) is Pearson correlation between the entire timecourses of pairs of brain regions. This correlation value is termed as functional connectivity (FC) and implicitly assumes that the underlying processes are stationary. Encoding FC as edge weights in graph models has been used to explore functional brain networks in terms of modularity/communities [2], which is indeed a property of functional organization in the brain [3].
However, recent evidence has started to acknowledge the dynamic nature of FC [4]. The most common technique to look into dynamic functional connectivity $(\mathrm{dFC})$ is based on a sliding-window approach; i.e., FC is computed only in a limited-time window that slides over the data. Different methods such as $k$-means clustering, singular value decomposition, and dictionary learning have been proposed to extract meaningful dFC patterns of brain states in healthy subjects or in specific patient groups $[5,6,7]$. However, there is still no method to explore changes in FC patterns during a full-length fMRI acquisition.

Here, we propose a new concept to extract traces of brain function by projecting $\mathrm{dFC}$ into a low-dimensional space where the interpretation of dynamics becomes easier to recognize. We leverage in particular the use of the graph Laplacian, to be sensitive to changes in network organization, and of Riemannian manifold properties, to define a rigorous measure of distance between graphs. The graph Laplacian matrix is often used to study modular organization $[8,9]$ and it has recently been proposed to explore dynamical process of network systems [10]. Moreover, the Laplacian matrix is positive semi-definite (PSD) and it can be combined with the Riemannian geometry [11]. Therefore, we characterize dissimilarity between dFC patterns using Riemannian metrics. At the same time, we discount accumulated distance over time to allow detection of recurrent patterns and obtain a measure of geodesic distance on the brain-state manifold. Finally, multi-dimensional scaling is used to project these distances into a lower-dimensional space allowing to visualize the trajectories.

We applied the proposed method to existing fMRI dataset of 15 healthy participants [12]. During fMRI scanning, resting states was alternated with different types of movie conditions (i.e., fearful, joyful, and neutral). The emotional content of the movies was shown to subtly influence the subsequent resting-state periods.

While our method is completely blind to the rest-movie paradigm and the movie conditions, the results show that (1) 
the extracted traces are consistent across subjects; (2) clear differences can be distinguished between resting state following different movie conditions. Our results further suggest that both the graph Laplacian and Riemannian distance are essential ingredients to let these meaningful traces emerge.

\section{MATERIALS \& METHODS}

\subsection{Dataset}

The dataset is composed by fifteen healthy participants, scanned with a Siemens 3T Trio TIM. Functional images were acquired in 2 scanning runs (2598 volumes in total) with the following parameters: repetition time (TR) $1100 \mathrm{~ms}$, echo time (TE) $27 \mathrm{~ms}$, flip angle 90, 21 axial slices, in-plane resolution of $3.75 \times 3.75 \mathrm{~mm}(64 \times 64$ matrix $)$, and slice thickness $4.2 \mathrm{~mm}$ with a $1.05 \mathrm{~mm}$ gap. We also acquired a field map and a high-resolution T1-weighted anatomical scan. The paradigm consisted of showing short nine movie excerpts (of three different types) interleaved with 90s resting periods. For the following analysis we consider only one scanning run using 1290 volumes. Further details on the dataset can be found in [12].

\subsection{Dynamic Functional Connectivity}

After basic preprocessing steps including realignment, normalization, and extracting regional timecourses using the AAL atlas [13, 14], we estimated dFC using pairwise Pearson correlation in sliding windows of length $\Delta t=20 \mathrm{TR}$ (22s) and with step size $s=5$ TR (5.5s) [15]. This led to a connectivity matrix for each window denoted as $W_{t}$. Concatenating all $W_{t}$ over the time results in a $(N \times N) \times T$ matrix for every subject, where $T$ is the number of windows and $N$ the number of brain regions. We then transformed and normalized all values in each window $W_{t}$ through Fisher $r$-to- $z$ transform.

Due to the fMRI acquisition protocol, we performed the above dFC analysis only during the sequence of the restingmovie paradigm, discarding the preparation sequence in between each resting-movie sequence as described in [12]. We applied this procedure to all subjects, which resulted in $T=$ $150 \mathrm{dFC}$ frames per subjects.

\subsection{Graph Laplacian and Modularity}

Although there are several methods taking into account the properties of the modularity matrix for graph partitioning [2, 16], we built our method upon the graph Laplacian. Spectral properties of the graph Laplacian are often combined with $k$ means clustering for community detection as relaxation of the Ncut problem [17]. In our method we computed the normalized Graph Laplacian for each temporal window as follow:

$$
L_{t}=D_{t}^{-1 / 2}\left(D_{t}-W_{t}\right) D_{t}^{-1 / 2},
$$

obtaining the dynamic Laplacian connectivity (dLC), where $D_{t}$ is the degree matrix of $W_{t}$ at each time $t$.

The graph Laplacian is PSD and it can be easily regularized to a positive definite matrix by the modification $\hat{L}_{t}=L_{t}+\gamma I$, where $\gamma>0$ is a regularization parameter and $I$ is the identity matrix [11]. In our settings we used $\gamma=10^{-9}$.

\subsection{Traces of Moment-to-Moment Fluctuations}

The key point to disentangle different brain states and to build the trace is the choice of the dissimilarity metric. Euclidean distance between $\mathrm{dFC}$ frames (after direct embedding of the upper-triangular part in a vector space) does not guarantee to correctly describe the dissimilarity between graphs, especially if a different modular organization has to be recognized. Regularized Laplacians $\hat{L}$ instead allow using Riemannian manifold properties.

We adopted the log-Euclidean distance between two positive definite matrices, which is geodesic and fast to compute [18]. Specifically, the distance between two timepoints $\hat{L}_{t_{i}}$ and $\hat{L}_{t_{j}}$ is obtained as

$$
d\left(\hat{L}_{t_{i}}, \hat{L}_{t_{j}}\right)=\left\|\log \left(\hat{L}_{t_{i}}\right)-\log \left(\hat{L}_{t_{j}}\right)\right\|_{F},
$$

with $\log (\cdot)$ denoting the principal matrix logarithm and \|\|$_{F}$ denoting the Frobenius matrix norm.

Eq. 2 does not consider the temporal dependencies between each frame and indeed, it does not allow to recognize recurring brain states since it implicitly models time linearly. However, whenever the distance between dFC frames for two timepoints is shorter than the average of the incremental distances for all successive timepoints in between them, we revert to the minimal distance:

$$
D\left(\hat{L}_{t_{i}}, \hat{L}_{t_{j}}\right)=\min \left(d\left(\hat{L}_{t_{i}}, \hat{L}_{t_{j}}\right), d_{\text {inc }}\left(\hat{L}_{t_{i}}, \hat{L}_{t_{j}}\right)\right),
$$

where

$$
d_{\text {inc }}\left(\hat{L}_{t_{i}}, \hat{L}_{t_{j}}\right)=\frac{1}{\left|t_{j}-t_{i}\right|} \sum_{k=\min (i, j)}^{\max (i, j)-1} d\left(\hat{L}_{t_{k}}, \hat{L}_{t_{k+1}}\right) .
$$

In other words, we propose a trade-off between $\mathrm{dFC}$ dissimilarity independent of time (Eq. (2)) and one that converges to an average distance as a function of time distance (Eq. (4)).

These dissimilarities are computed for all pairwise combinations of timepoints (per subject) generating the distance matrix $D$ (dimension $T \times T$ ). Non-metric multidimensional scaling (nMDS) ${ }^{1}$ is applied to reduce the dissimilarity matrix $D$ into a lower dimensional space $K$ and the procrustes analysis is used to adapt the shapes (i.e. the traces in our setting) to a reference space. We applied it to the traces of the $\mathrm{dFC}$, aiming at aligning all the trajectories so as to highlight the agreement across the subjects. As a reference space we selected a

\footnotetext{
${ }^{1}$ Implementation in Matlab R2014a.
} 
random subject and we iterated the procrustes analysis till we obtained a good fit based on the sum of squared errors (generally after few iterations). Finally, for visualization purposes, we considered only the first 3 dimensions that were used to plot the traces of brain activity.

\section{RESULTS \& CONCLUSION}

Fig. 1 shows qualitative results of the trace obtained with the entire pipeline for a representative single subject using both our new distance (Eq. 3,4) and classical Euclidean distance. We replaced the geodesic distance with euclidean one in (Eq. 3, 4) applying it both on the graph Laplacian and modularity matrix of the $\mathrm{dFC}$ frames. The projection of the distance matrix obtained with Euclidean distance does not lead to a clear trace that is meaningful in terms of temporal relationships and/or clustering of brain states. In particular, the point clouds do not show a separation between resting and movie periods. The proposed method, on the contrary, let us identify both clusters that represent resting state following different movie conditions, as well as a clear trace that respects time. We also performed a quantitative evaluation of nMDS. Kruskal Stress [19] is commonly used to evaluate the performance of multidimensional scaling algorithms. Values grater than 0.2 indicate a bad fit while values lower than 0.05 indicate a good fit of the original data in the lower dimensional space. We computed the Kruskal Stress for each subject and we found an average stress of $0.009 \pm 0.001$ with $K=97$, where $K$ is the dimension of the subspace which better approximates the dFC. Also the analysis with the euclidean distance gives similar results in term of Kruskal Stress but more dimensions are needed to reach the best fitting of the input data $\left(K_{\text {lap }}=123, K_{\text {mod }}=124\right)$. It denotes that our proposed metric combined with graph Laplacian works better than the euclidean one.

Fig. 2 shows the traces for four different subjects after the procrustes analysis. We adopted the procrustes analysis to realign different traces on a reference space, mostly to ease the comprehension of rs-fMRI trajectory. The coloring of the points indicates the temporal sequence. It reveals a significant level of resemblance between subjects: moviewatching frames are almost always limited to points close to the center, while resting-state points are much more spread out in directions related to the preceding movie conditions. We found that this behaviour of the traces is highly reproducible across subjects, which shows that the resting-state brain is functionally reorganized according to the emotional state induced by the movies (fearful, joyful, neutral). It is also surprising that the movie frames are projected close to the center of the space, suggesting that passive movie-watching has a less richer structure than the spontaneous (but emotionally induced) resting-state periods that follow.

These results corroborate and further deepen the findings reported in [12], where specific hypothesis-driven FC analy- sis of short time segments and regions revealed already emotional induction of resting state by the preceding movie condition. Our method is blind to the structure of the experimental paradigm and considers whole-brain connectivity. More detailed analysis should further investigate which brain connectivity drives the traces to the distinct configurations that we observe.

In summary, we proposed a novel concept to trace and study dFC. The obtained results are a proof-of-principle for the combined use of the graph Laplacian to measure modular organization and of Riemannian distance to assess dissimilarity of dFC over time. This allowed us to distinguish and characterize relevant brain states.

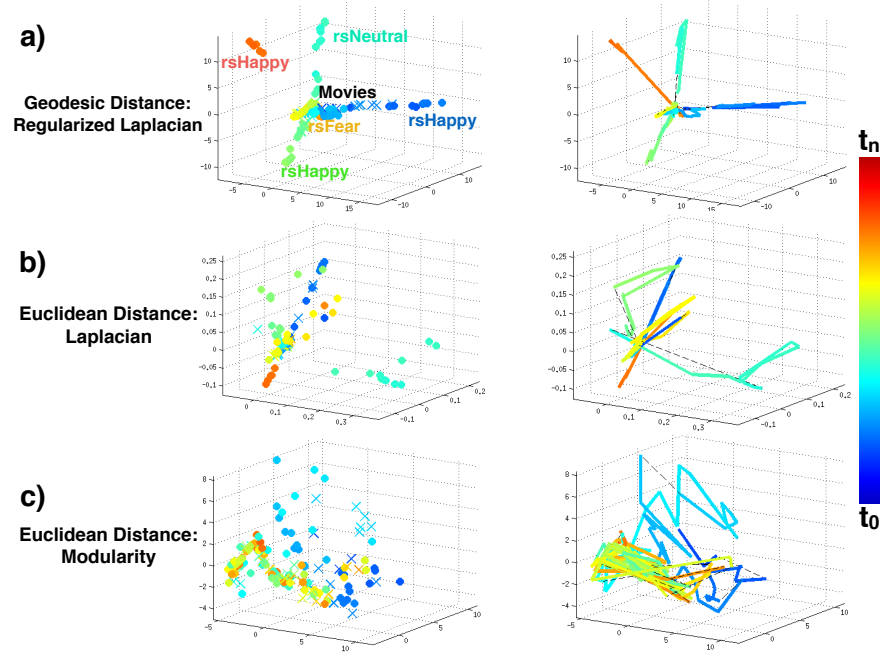

Fig. 1. Traces in 3-D obtained for a single subject and different distance measures. a: Proposed Riemannian distance on the graph Laplacian; b: Euclidean distance on the graph Laplacian; c: Euclidean distance on the modularity matrix. Each timepoint is indicated by a cross (movie) or a dot (rest). Dots are colored according to time. Clusters in a) correspond to different resting periods according to the preceding movie conditions (i.e., labelled rsHappy, rsFear, rsNeutral).

\section{ACKNOWLEDGEMENTS}

This work was supported in part by the $\mathrm{PhD}$ program of University of Genova, in part by the Swiss National Science Foundation (grants PP00P2-146318 and 205321-163376), in part by the Center for Biomedical Imaging (CIBM). The authors are also grateful to H. Eryilmaz, S. Schwartz, P. Vuilleumier for making available the dataset of [12].

\section{REFERENCES}

[1] M. P. Van Den Heuvel and H. E. H. Pol, "Exploring the brain network: a review on resting-state fmri functional connectiv- 

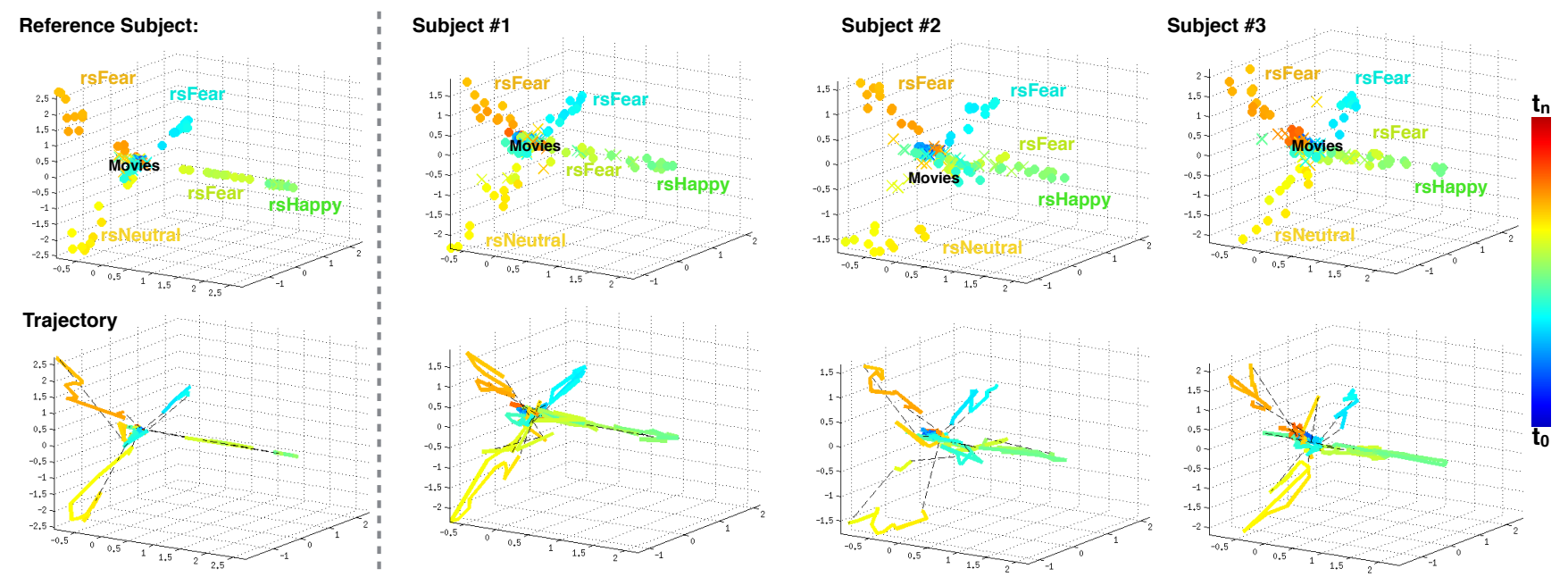

Fig. 2. Traces in 3-D of four random subjects. On the left the subject used as a reference for the procrustes analysis.

ity," European Neuropsychopharmacology, vol. 20, no. 8, pp. 519-534, 2010.

[2] M. Girvan and M. E. Newman, "Community structure in social and biological networks," Proceedings of the national academy of sciences, vol. 99, no. 12, pp. 7821-7826, 2002.

[3] D. Meunier, R. Lambiotte, and E. T. Bullmore, "Modular and hierarchically modular organization of brain networks," Frontiers in neuroscience, vol. 4, 2010.

[4] C. Chang and G. H. Glover, "Time-frequency dynamics of resting-state brain connectivity measured with fmri," Neuroimage, vol. 50, no. 1, pp. 81-98, 2010.

[5] E. A. Allen, E. Damaraju, S. M. Plis, E. B. Erhardt, T. Eichele, and V. D. Calhoun, "Tracking whole-brain connectivity dynamics in the resting state," Cerebral cortex, p. bhs352, 2012.

[6] N. Leonardi, J. Richiardi, M. Gschwind, S. Simioni, J.-M. Annoni, M. Schluep, P. Vuilleumier, and D. Van De Ville, "Principal components of functional connectivity: a new approach to study dynamic brain connectivity during rest," NeuroImage, vol. 83, pp. 937-950, 2013.

[7] N. Leonardi, W. Shirer, M. Greicius, and D. Van De Ville, "Disentangling dynamic networks: Separated and joint expressions of functional connectivity patterns in time," Human Brain Mapping, vol. 35, no. 12, pp. 5984-5995, December 2014.

[8] L. Dodero, A. Gozzi, A. Liska, V. Murino, and D. Sona, "Group-wise functional community detection through joint laplacian diagonalization," in Medical Image Computing and Computer-Assisted Intervention-MICCAI 2014, pp. 708-715. Springer, 2014.

[9] M. Van Den Heuvel, R. Mandl, and H. Hulshoff Pol, "Normalized cut group clustering of resting-state fmri data," PloS one, vol. 3, no. 4, pp. e2001, 2008.

[10] J.-C. Delvenne, R. Lambiotte, and L. E. Rocha, "Diffusion on networked systems is a question of time or structure," Nature communications, vol. 6, 2015.
[11] L. Dodero, H. Q. Minh, M. S. Biagio, V. Murino, and D. Sona, "Kernel-based classification for brain connectivity graphs on the riemannian manifold of positive definite matrices," in Biomedical Imaging (ISBI), 2015 IEEE 12th International Symposium on. IEEE, 2015, pp. 42-45.

[12] H. Eryilmaz, D. Van De Ville, S. Schwartz, and P. Vuilleumier, "Impact of transient emotions on functional connectivity during subsequent resting state: a wavelet correlation approach," Neuroimage, vol. 54, no. 3, pp. 2481-2491, 2011.

[13] J. Richiardi, H. Eryilmaz, S. Schwartz, P. Vuilleumier, and D. Van De Ville, "Decoding brain states from fMRI connectivity graphs," NeuroImage, vol. 56, no. 2, pp. 616-626, 2011.

[14] N. Tzourio-Mazoyer, B. Landeau, D. Papathanassiou, F. Crivello, O. Etard, N. Delcroix, B. Mazoyer, and M. Joliot, "Automated anatomical labeling of activations in spm using a macroscopic anatomical parcellation of the mni mri single-subject brain," Neuroimage, vol. 15, no. 1, pp. 273-289, 2002.

[15] N. Leonardi and D. Van De Ville, "On spurious and real fluctuations of dynamic functional connectivity during rest," Neuroimage, vol. 104, pp. 430-436, 2015.

[16] D. S. Bassett, N. F. Wymbs, M. A. Porter, P. J. Mucha, J. M. Carlson, and S. T. Grafton, "Dynamic reconfiguration of human brain networks during learning," Proceedings of the National Academy of Sciences, vol. 108, no. 18, pp. 7641-7646, 2011.

[17] J. Shi and J. Malik, "Normalized cuts and image segmentation," Pattern Analysis and Machine Intelligence, IEEE Transactions on, vol. 22, no. 8, pp. 888-905, 2000.

[18] V. Arsigny, P. Fillard, X. Pennec, and N. Ayache, "Logeuclidean metrics for fast and simple calculus on diffusion tensors," Magnetic resonance in medicine, vol. 56, no. 2, pp. 411421, 2006.

[19] J. B. Kruskal, "Multidimensional scaling by optimizing goodness of fit to a nonmetric hypothesis," Psychometrika, vol. 29, no. 1, pp. 1-27, 1964. 